\title{
The effect of adjunctive nutraceuticals in bipolar disorder: A systematic review of randomized placebo-controlled trials
}

\author{
Laura Fusar-Poli, Teresa Surace, Antonio Vanella, Valeria Meo, Federica Patania, \\ Rosaria Furnari, Maria Salvina Signorelli, Eugenio Aguglia \\ Department of Clinical and Experimental Medicine, Psychiatry Unit, University of Catania, Italy
}

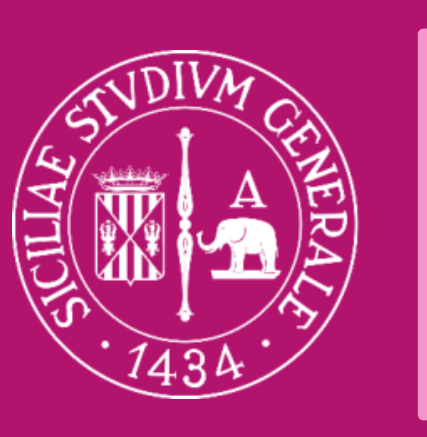

We performed a systematic search following the PRISMA guidelines, from inception to February 2019. We included all original articles written in English which met the following criteria:

(1) Participants: individuals with bipolar disorder (type I or type II), mania or schizoaffective disorder, bipolar type, diagnosed by a clinician according to international valid diagnostic criteria (i.e. DSM or ICD).

(2) Intervention: nutraceuticals of any type, dosage and form of administration (i.e. omega 3 fatty acids, vitamins, minerals, amino acids, $\mathrm{N}$-acetyl cysteine, probiotics, etc), in combination with standard care.

(3) Comparison: placebo plus standard care.

(4) Outcomes: depressive symptoms or manic symptoms, evaluated through standardized measures, clinical global impression, functioning, adverse events, other outcomes.

(5) Study design: randomized controlled trials, both parallel and crossover.

The PRISMA flow chart of the study selection process has been reported in Figure 1.

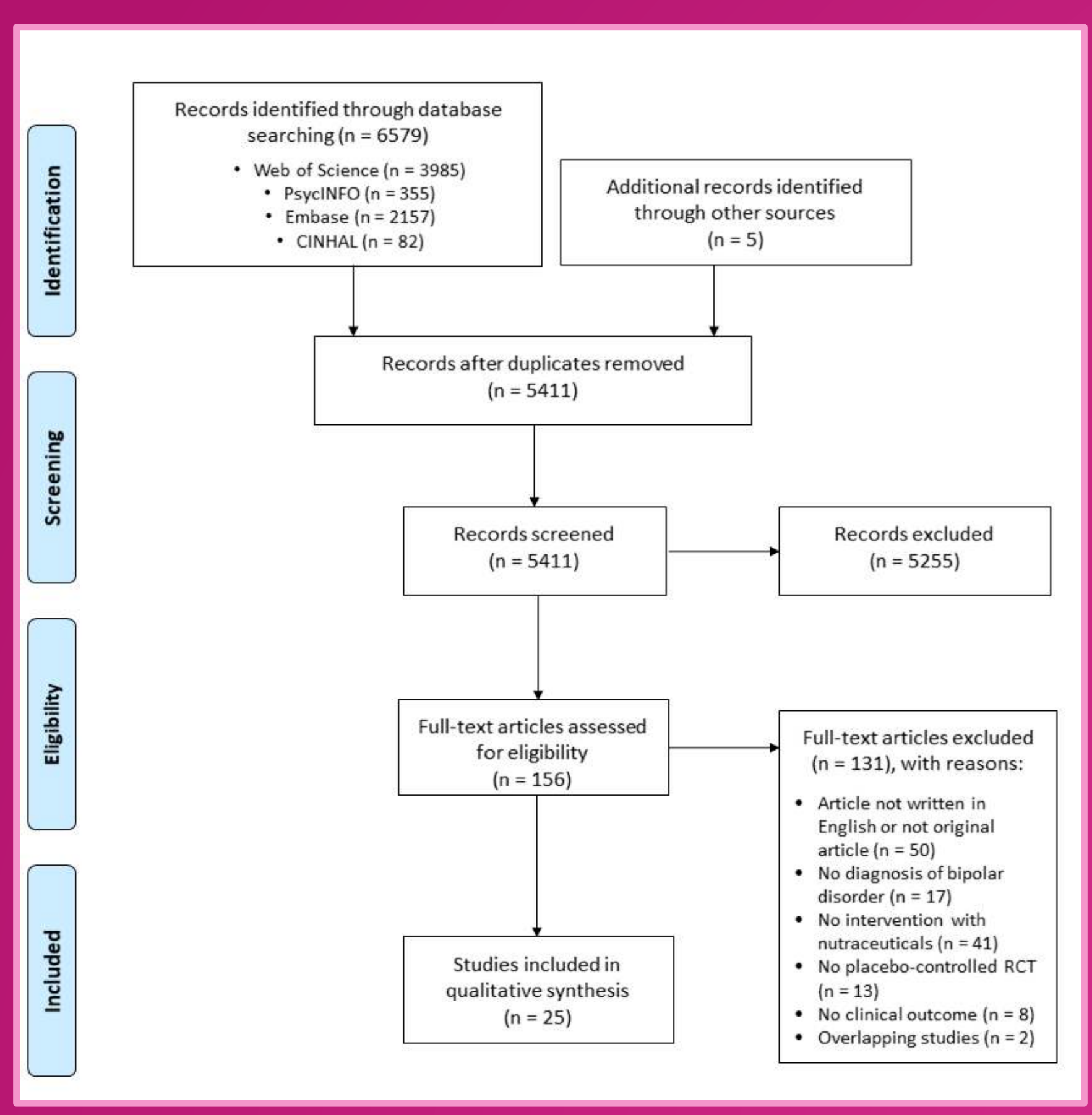

Full reference

Fusar-Poli, L., Surace, T., Vanella, A., Meo, V., Patania, F., Furnari, R.,

Signorelli M.S., Aguglia, E. (2019). The effect of adjunctive nutraceuticals in bipolar disorder: A systematic review of randomized placebocontrolled trials. Journal of Affective Disorders, 252, 334-349.

After identifying 6584 potentially relevant publications, we finally included $\mathbf{2 5}$ studies, testing the following nutraceuticals:

- Fatty acids (6)

- Micronutrients (7)

- Amino acids (7)

- Probiotics (1)

- Combination of different nutraceuticals (4)

Even if some compounds have shown promising results (i.e. fatty acids and $\mathrm{N}$-acetyl cysteine for depression, amino acid drinks and folic acid for mania), the majority of nutraceuticals did not cause significant improvements in comparison to placebo. We could not perform a meta-analysis due to the high heterogeneity of trials, which were also affected by some methodological caveats.

The risk of bias of the included studies has been reported in Figure 2.

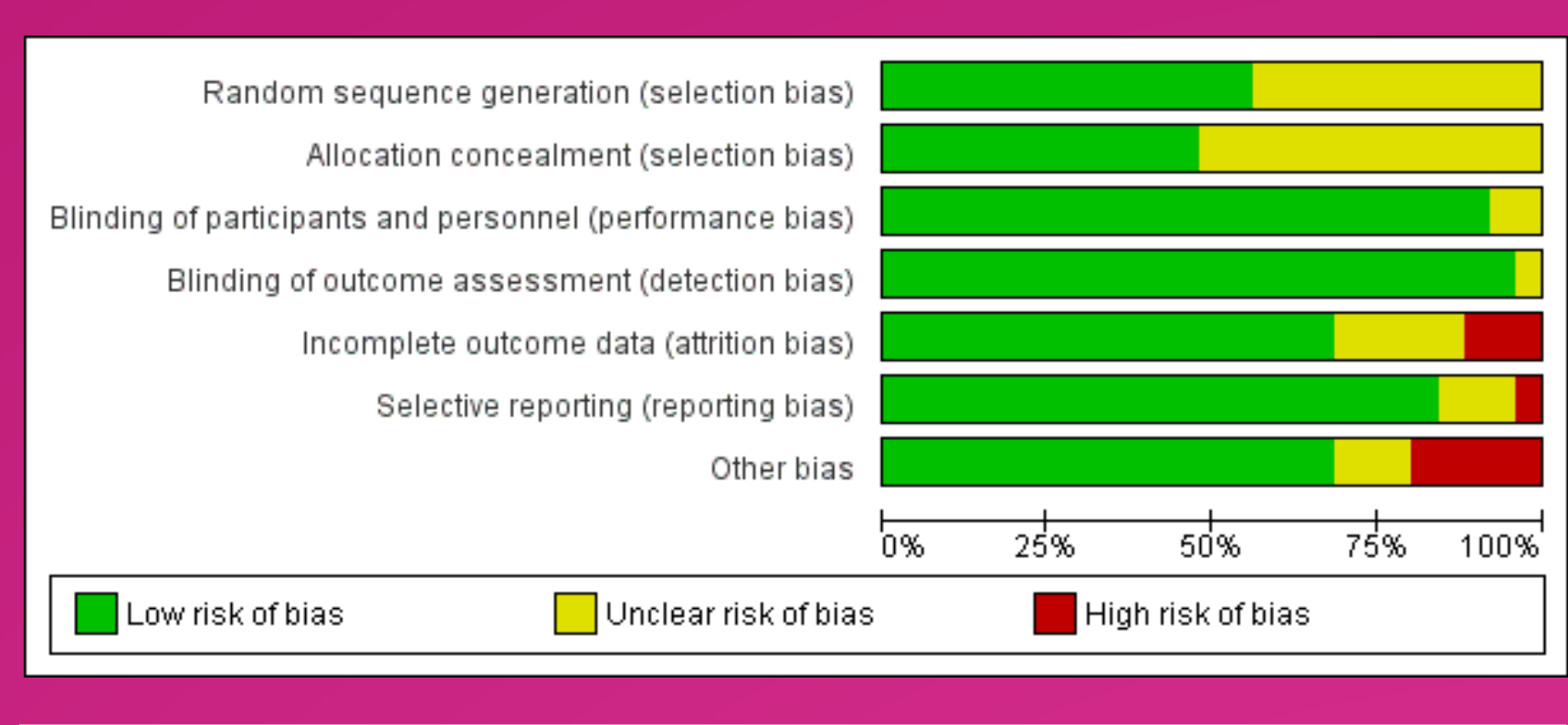

\section{CONCLUSION}

The present systematic review revealed inconsistent results about the effectiveness of nutraceuticals on symptoms of BD. Some of them seem to be potentially useful, but significant results often rely on single or few studies. However, nutraceuticals appeared generally safe and free of important side effects.

More clinical trials are needed to better elucidate the role of nutraceuticals in treating core symptoms of BD. It is important for future researchers to better characterize and, possibly, harmonize the samples recruited in trials examining the role of nutraceuticals in BD. Additionally, all concomitant conditions potentially influencing the response to treatment (i.e, baseline nutrient levels, dietary regimen) should be clearly explained.

Finally, the role of promising substances not included in this review (e.g. saffron, curcumin, etc.) should be tested by researchers in future investigations. 